Table 1 Perception of disease and treatment and adherence level

\begin{tabular}{|c|c|c|c|c|}
\hline & \multicolumn{3}{|l|}{ Adherence level } & \multirow[b]{2}{*}{ p Value } \\
\hline & $\operatorname{High}(n=84)$ & Medium $(n=55)$ & Low $(n=75)$ & \\
\hline \multicolumn{5}{|c|}{ Perception of disease (No (\%)) } \\
\hline Enslaving & $30(35.7)$ & $21(38.2)$ & $20(26.7)$ & 0.3 \\
\hline Cruel & $26(31.0)$ & $14(25.4)$ & $23(30.7)$ & 0.7 \\
\hline Threatening & $23(27.4)$ & $17(30.9)$ & $22(29.3)$ & 0.9 \\
\hline Invasive & $24(28.6)$ & $13(23.6)$ & $20(26.7)$ & 0.8 \\
\hline Constructive & $12(14.3)$ & $7(12.7)$ & $17(22.7)$ & 0.23 \\
\hline \multicolumn{5}{|c|}{ Perception of therapy (No (\%)) } \\
\hline Protective & $42(50.0)$ & $21(38.2)$ & $25(33.3)$ & 0.09 \\
\hline Reliable & $37(44.1)$ & $28(50.9)$ & $20(26.7)$ & 0.01 \\
\hline Allied & $31(36.9)$ & $23(41.8)$ & $28(37.3)$ & 0.82 \\
\hline Exigent & $25(29.8)$ & $18(32.7)$ & $29(38.7)$ & 0.49 \\
\hline Enslaving & $17(20.2)$ & $14(25.4)$ & $29(38.7)$ & 0.03 \\
\hline
\end{tabular}

${ }^{\star} \chi^{2}$ adjusted for age, sex, and clinical status (symptomatic and asymptomatic).

danavir $(25.2 \%)$ /zidovudine, lamivudine, indinavir $(10.7 \%) /$ stavudine, lamivudine $(8.4 \%) /$ stavudine, lamivudine, saquinavir $(7.0 \%) /$ ziduvudine, lamivudine $(6.5 \%)$ /ziduvudine, lamivudine, saquinavir $(6.0 \%)$.

Most respondents perceive HIV disease as enslaving $(33.2 \%)$, cruel $(29.4 \%)$, and threatening $(29 \%)$. Among the respondents' choices, the connotation "constructive" was indicated by 36 subjects $(16.8 \%)$. With regard to the perception of therapy, most patients perceived therapy as protective ( 88 subjects).

Table 1 shows adherence level according to perception of disease and therapy.

The perception of disease was not associated with adherence level. However, the perception of therapy as protective or reliable was associated with higher levels of adherence, and the perception of therapy as enslaving with lower ones. These findings were confirmed after taking into account potential confounding factors (including sex, age, and clinical status) and, in particular, the perception of therapy was an independent factor of adherence in strata of different perception of the disease (data not shown).

In conclusion, the patients' attitudes toward therapy emerge as important in ensuring high adherence levels. As correct adherence to treatment is a necessary condition in order to obtain therapy efficacy, these findings suggest it would be useful to consider perception of therapy by the doctor in patients' management.

We thank the patients for their participation; A Gazzani for data analysis assistance, directors of Infectious Diseases Clinics where we recruited our patients and their collaborators: Drs A Cargnel, ${ }^{\star} \mathrm{P}$ piva, ${ }^{\star}$ F Mazzotta, $\dagger$ M Di Pietro, $\dagger$ G Carosi, $\ddagger S$

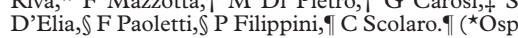
Sacco, Milan; †Osp SM Annunziata, Florence; ¥University of Brescia; 』University 'Sapienza' Rome; $\uparrow$ Osp "Gesù e Maria”, University of Naples.) We $\uparrow$ Osp "Gesu e Maria", University of Naples.)
also thank E Recchia, research assistance, We also thank E Recchia, research assistance,
LILA (Italian League for the fight against AIDS,

National Research Center, Milan).
This study was partially granted by the Italian minister of health.

M MARTINI P NASTA

LILA Lega Italiana per la Lotta contro l'AIDS (Italian League for the fight against AIDS), National Research Centre, Milan, Italy E RICCI

F PARAZZIN

Istituto di Ricerche Farmacologiche, "Mario Negri", Milan, Italy

V AGNOLETTO

LILA Lega Italiana per la Lotta contro l'AIDS, Via Rogoredo, 41, 20138, Milan, Italy

Correspondence to: Dr V Agnoletto

1 Chesney MA, Morin M, Sherr L. Adherence to HIV combination therapy. Soc Sci Med 2000;50:1599-605.
2 Broers B, Morabia A, Hirshel B. A cohort study of drug users compliance with zidovudine treatment. Arch Intern Med 1994;154:1121-7. 3 Mehta S, Moore RD, Graham NMH. Potential factors affecting adherence with HIV therapy. AIDS 1997;11:1665-70.

Accepted for publication 12 October 2000

\section{The power of information and contraceptive choice in a family planning setting in Mexico}

EDITOR, - In the article by Lazcano Ponce et $a l,{ }^{1}$ the proportion of women receiving IUDs fell in the intervention arm. As the authors state, most methods of contraception do not protect against STI acquisition. Most of the women choosing not to use IUDs in this trial probably selected oral contraceptives, another non-barrier method. These women, too, would be unprotected against STI, so the benefit of this intervention is unclear. In addition, most other methods of contraception have higher pregnancy failure rates than IUDs; thus, women deterred from IUDs in this trial may have had more unplanned pregnancies, with their attendant risks and cost, as a result of the intervention.

Fully informed choice for all family planning clients is an estimable standard of care. But the authors' unstated assumption seems to be that too many women at risk of STI receive IUDs for family planning. ${ }^{1}$ While IUDs cause a transient increase in the risk of pelvic inflammatory disease, they have no documented adverse effect on STI acquisition or ascent thereafter. ${ }^{2}$ IUDs offer safe, effective, affordable long term contraception. Given the low prevalence of cervical infection among family planning clinic attenders in this trial ${ }^{1}$ and others, we need guidelines to "rule in" the great majority of women who are uninfected, rather than inappropriately label large numbers of women ineligible for IUD use.

PAUL FELDBLUM Family Health International, Clinical Research Department, PO Box 13950, Research Triangle Park, NC 27709, USA

1 Lazcano Ponce EC, Sloan NL, Winikoff B, et al. The power of information and contraceptive choice in a family planning setting in Mexico. choice in a family planning setting
Sex Transm Inf 2000;76:277-81.

2 Grimes DA. Intrauterine device and uppergenital-tract infection. Lancet 2000;356:101319

Accepted for publication 12 October 2000

\section{AIDS and globalisation}

EDIToR,-I commend Shamanesh et $a l^{1}$ for their searching and informed account of the impact of globalisation on the world AIDS problem. Revisiting Alma Ata 1978: the existence of gross inequalities between advantaged and disadvantaged peoples is "politically, socially and economically" unacceptable. Twenty two years on, are we closer to the ideal of "health for all" or further away. When will we learn?

MIKE GRIFFITHS
SHO Public Health/Non-Principal GP,
West Midlands, UK
michael.griffiths@dudley-ha.wmids.nhs.uk
1 Shahmanesh M, Shahmanesh M, Shahmanesh
R. AIDS and globalisation. Sex Transm Inf
2000;76:154-5.

\section{WSW status needs further definition}

EDITOR,-Fether et al present a very interesting case-control study on STIs in women who have sex with women (WSW). ${ }^{1}$ This was not a community based sample and thus prone to selection bias. In order to appreciate the results in full it would help to know how cases and controls were identified and how controls were selected. Bisexual or homosexual orientation may be difficult to disclose even in a sympathetic and non-judgmental setting. Studies using self reported sexual orientation to determine case or control status will always have a degree of differential misclassification. It is likely that WSW who volunteer this information differ not only from women who do not have sex with women but also from WSW who do not volunteer the information but admit it when prompted, and from those who do not admit it even when prompted. Without this information it is difficult to determine the importance of various prevalences quoted in the paper. All I learn from this paper at present is that women who have sex with women also take other risks.

RUDIGER PITTROF

St George's Hospital, London, UK

rudiger_pittrof@hotmail.com

1 Fethers K, Marks C, Mindel A, et al. Sexually transmitted infections and risk behaviours in women who have sex with women. Sex Transm Inf 2000;76:345-9.

\section{BOOK REVIEWS}

A Physician's Guide to Clinical Forensic Medicine. Ed Margaret M Stark. Pp 326; $\$ 79.95$. Totowa: Humana Press, 2000. ISBN 0-896-03742-8.

Forensic examination of those alleging sexual assault can be appropriately provided within sexual health services, but requires additional skills. The examinations are lengthy, detailed, frequently nocturnal, and often lead to an appearance at the Old Bailey. A distressed individual will need to be examined from head to toe, with every injury described, measured, and drawn. The correct samples must be taken, timed, labelled and bagged with due attention to the chain of evidence. Subsequently the doctor may need to interpret the findings for the police, write a detailed statement, and withstand the adversarial setting of the court.

A Physician's Guide to Clinical Forensic Medicine covers areas relevant to general forensic 